\title{
Assessment of Left Ventricular Function by Global Longitudinal Strain in Patients with non-ST Elevation Myocardial Infarction: Comparative Study with Conventional Method
}

\author{
Sena Mohamed S. Ahmad, Elsayed Mohamed Farag, Mohammad Abdalla Eltahlawi, Alaa Elsayed Salama \\ Department of Cardiology, Faculty of Medicine, Zagazig University, Egypt \\ *Corresponding author: Sena M. S. Ahmad, Mobile: (+20)10467874428, Email: sana.sultan2030@ gmail.com
}

\begin{abstract}
Background: Left ventricular ejection fraction (LVEF) is a strong prognostic parameter in patients with heart disease. The assessment of global longitudinal strain (GLS) from speckle-tracking analysis of 2-dimensional echocardiography has become a clinically feasible alternative to LVEF for the measurement of myocardial function.

Objective: The aim of the current work was to compare between GLS speckle tracking echocardiography and Simpson's biplane methods for assessment of left ventricular function in non-STEMI patients.

Patients and methods: This study was carried out in Cardiology Department, Faculty of Medicine, Zagazig University on 44 non-ST-elevation myocardial infarction (NSTEMI) patients scheduled for assessment of Left ventricular function by GLS and biplane Simpson method.

Result: Mean systolic blood pressure was $122.05 \pm 18.37 \mathrm{mmHg}$. No statistically significant difference in systolic blood pressure was found across GLS groups $(\mathrm{P}=0.17)$. Mean diastolic blood pressure was $73.41 \pm 12.00 \mathrm{mmHg}$. No statistically significant difference in diastolic blood pressure was found across GLS groups $(\mathrm{P}=0.35)$. Mean heart rate was $68.84 \pm 7.13 \mathrm{bpm}$, and a statistically significant difference was found in heart rate across GLS groups $(\mathrm{P}=0.039)$. Conclusion: It could be concluded that GLS speckle tracking echocardiography and Simpson's biplane methods can be used as alternative different parameters for assessment of left ventricular function in non-STEMI patients.
\end{abstract}

Keywords: Left Ventricular Function; Speckle-Tracking; Global Longitudinal Strain

\section{INTRODUCTION}

The noninvasive assessment of ventricular function remains central to modern cardiology. The volume-based measurement of left ventricular ejection fraction (LVEF) is fundamentally different from direct measurement of myocardial motion by tissue Doppler imaging and myocardial deformation, and the reliability and precision of these measurements are also different (1). In the era of precision medicine, patient-specific measurements are used to make decisions about therapies in individual patients, as well as guidance across patient populations. Moreover, the current era is also marked by the emergence of heart failure with preserved ejection fraction (HFpEF) in which ejection fraction $(\mathrm{EF})$ is not useful prognostically as the predominant form of heart failure (HF) ${ }^{(2)}$. Acute coronary syndromes (ACS) continue to be a major cause of morbidity and mortality. Data from the United States and Europe have reported a decrease in the incidence of ST-elevation myocardial infarction (STEMI) with an increase in NSTEMI in the past decade ${ }^{(3)}$.

Left ventricular ejection fraction (LVEF) is the established method for evaluation of LV systolic function and can be measured by a number of imaging modalities. LVEF by echocardiography has been regarded as a cornerstone in the prediction of outcome and is the most widely available method for evaluation of LV function ${ }^{(4)}$.

It is a vital measurement when determining whether patients benefit from an implantable cardioverter-defibrillator (ICD) or cardiac resynchronization therapy (CRT) ${ }^{(5)}$.

In addition, LVEF is used to define systolic heart failure and has a great impact on the selection of medical treatment ${ }^{(\mathbf{6})}$.

Several echocardiographic methods have been used to measure LVEF but at present, the Simpson's biplane method is most widely used ${ }^{(7)}$. Determining LVEF by echocardiography is associated with a high level of inter-observer variability, which to a certain degree can be improved using contrast enhanced echocardiography and 3D echocardiography. Reliability of LVEF depends on image quality and in particular the ability to visualize the endocardial border (8). Strain by speckle tracking echocardiography is a technique that utilizes 2-dimensional gray scale images to evaluate both global and regional function of the left ventricle. Peak global longitudinal strain (GLS) may be used to measure systolic function. Previous studies have shown that GLS may both diagnose and exclude acute coronary heart disease ${ }^{(\boldsymbol{9})}$.

In addition, GLS has better intra- and interobserver reproducibility in post hoc analysis compared to LVEF ${ }^{\left({ }^{10}\right)}$. Furthermore, GLS may be analyzed in a majority of patients with good feasibility and may be measured as fast as LVE ${ }^{(11)}$.

The aim of the study was to compare between GLS speckle tracking echocardiography and Simpson's biplane methods for assessment of left ventricular function in non STEMI patients. 


\section{PATIENTS AND METHODS}

This prospective cohort study included a total of 44 NSTEMI patients scheduled for assessment of left ventricular function by global longitudinal strain (GLS) and biplane Simpson method, attending at Cardiology Department; faculty of Medicine, Zagazig University Hospitals.

Thirty-two patients were males, and 12 patients were females. Thirty-two patients were $<56$ years and 12 patients were $\geq 56$ years.

Inclusion criteria: non-STEMI confirmed by ECG and cardiac enzymes.

Exclusion criteria: Pregnant females, severe valvular dysfunction cardiomyopathy, patients on

chemotherapy and anemia.

\section{All patients were subjected to:}

1) Complete history taking: Including name, age, sex, episode of acute chest pain lasting at least 10 min., special habits (especially smoking), menstrual state, history of drug intake or previous hospital admission with special consideration to history of risk factors of ischemic heart disease (especially hypertension, diabetes mellitus), and comorbid conditions.

2) Thorough physical examination: with special emphasis on pulse rate and rhythm to exclude arrhythmia, blood pressure (systolic \& diastolic).

3) Electrocardiographic examination: 12- lead Electrocardiograms (ECGs) were performed on admission and repeated every day during hospitalization stay. They were evaluated by experienced cardiologists and were considered to be abnormal in the presence of $\mathrm{N} 1 \mathrm{~mm}$ ST depression or T-wave inversion in at least two consecutive leads.

4) Laboratory investigations: Troponin I (TnI) assays were performed on admission and at 3 hours. Elevated troponin I was based on at least one assay above the upper limit of normal (99th percentile) defined by the laboratory $(\geq 100$ $\mathrm{pg} / \mathrm{mL})$. HbA1c was also done.

5) Conventional Transthoracic EchoDoppler study: Transthoracic echocardiographic examination was performed in the hour following the admission to the acute coronary care unit, and prior to coronary angiography, using a ViNNO8 Ultrasound device machine with S1-6P single transducer (VINNO Company, chine). The device software is 1.9 .6 based on RF technology. Images were taken while the patient in supine or in the left lateral position utilizing, apical 4 and apical 2 chamber views. Recordings and calculations of different parameters were performed according to the recommendations of the American Society of Echocardiography.
The following measures were stressed upon and selected for analysis:

\section{I- Left ventricular systolic function:}

A- Ejection fraction (EF \%): The ejection fraction was calculated from apical 4- and 2-chamber views with Simpson's method. End-diastolic and end-systolic endocardial borders were traced manually on frozen 2D images obtained from the apical two- and four-chamber views to derive end-diastolic volume (EDV) and endsystolic volume (ESV). The LV EF was calculated according to the formula. It is calculated also from the formula: $\quad \mathbf{E F}=\underline{\mathbf{E D V}-\mathbf{E S V}} \times \mathbf{1 0 0}$

$$
\text { EDV }
$$

B- Global Longitudinal Strain (GLS): Lagrangian strain is defined as the change in length of an object within a certain direction relative to its baseline length: Strain $\%=(\mathrm{L} 1-\mathrm{L} 0) / \mathrm{LO}$. The published values of GLS vary considerably from $-15.9 \%$ to $-22.1 \%$.The peak negative systolic longitudinal strain was assessed in all 17 longitudinal LV segments and the segmental values were averaged to give the global longitudinal strain (GLS). The reproducibility of the echocardiographic analyses was evaluated by determining the intraclass correlations for intra-observer and inter-observer variability. All patients are following up for any complication or MACE (Major Adverse Cardiac Events) during in hospital stay. The patients were finally classified in three groups according to GLS result.

\section{Ethical consent:}

An approval of the study was obtained from Zagazig University academic and ethical committee. Every patient signed an informed written consent for acceptance of the operation. This work has been carried out in accordance with The Code of Ethics of the World Medical Association (Declaration of Helsinki) for studies involving humans.

\section{Statistical analysis:}

Statistical presentation and analysis of the present study was conducted, using the mean, standard deviation, paired (T) test, Mann-Whitney test and analysis of variance [ANOVA] test. Continuous variables are summarized as mean $\pm \mathrm{SD}$. Categorical variables were compared by Chi-square while paired-t test was used to compare continuous variables between groups. All was calculated SPSS version 12 software program (12). The threshold of significance is fixed at 5\% level (P value) Significance is detected according to $\mathrm{P}$-value as follow: $\mathrm{P}>0.05$ Non-significant, $\mathrm{P}<0.05$ significant and $\mathrm{P}<0.001$ highly significant

\section{RESULTS}

In the present study, $73 \%$ of the patients were male and $27 \%$ were female. There was a statistically significant difference in gender across GLS groups $(\mathrm{P}=$ 0.004) (Table 1). Mean patient age was $50.32 \pm 10.73$ years. There was a statistically significant difference in age across GLS groups $(\mathrm{P}=0.02)$ (Table 2). 
About $36 \%$ of patients had diabetes mellitus, and a statistically significant association was found between presence of diabetes and GLS score $(\mathrm{P}<0.001)$. Mean HbA1c in diabetic patients was $8.19 \pm 1.14 \%$ (Table 3).

Mean systolic blood pressure was $122.05 \pm 18.37 \mathrm{mmHg}$. No statistically significant difference in systolic blood pressure was found across GLS groups $(\mathrm{P}=0.17)$. Mean diastolic blood pressure was $73.41 \pm 12.00 \mathrm{mmHg}$. No statistically significant difference in diastolic blood pressure was found across GLS groups $(\mathrm{P}=0.35)$ (Table 4).

Table (1): Patients' gender in relation to GLS groups

\begin{tabular}{|c|c|c|c|c|c|}
\hline Variable & $\begin{array}{l}\text { Overall, } \\
\mathrm{N}=\mathbf{4 4}^{1}\end{array}$ & $\begin{array}{c}\text { GLS higher than - } \\
10 \& \text { less than }-16, \\
\mathrm{~N}=17^{1}\end{array}$ & $\begin{array}{c}\text { GLS higher } \\
\text { than }-16, \\
N=1^{1} \\
\end{array}$ & $\begin{array}{c}\text { GLS less than - } \\
10, \\
N=12^{1} \\
\end{array}$ & p-value ${ }^{2}$ \\
\hline Gender & & & & & 0.004 \\
\hline Female & $12(27 \%)$ & $6(35 \%)$ & $0(0 \%)$ & $6(50 \%)$ & \\
\hline Male & $32(73 \%)$ & $11(65 \%)$ & $15(100 \%)$ & $6(50 \%)$ & \\
\hline
\end{tabular}

Table (2): Difference in patients' age (years) across GLS groups

\begin{tabular}{|c|c|c|c|c|c|}
\hline Variable & $\begin{array}{l}\text { Overall, } \\
N=44^{1}\end{array}$ & $\begin{array}{l}\text { GLS higher than - } \\
10 \& \text { less than }-16, \\
N=17\end{array}$ & $\begin{array}{c}\text { GLS higher } \\
\text { than }-16, \\
N=15\end{array}$ & $\begin{array}{c}\text { GLS less than - } \\
10, \\
\mathrm{~N}=12\end{array}$ & p-value ${ }^{2}$ \\
\hline Patient age & & & & & 0.020 \\
\hline Mean \pm SD & $50.32 \pm 10.73$ & $50.41 \pm 10.53$ & $45.53 \pm 11.17$ & $56.17 \pm 7.85$ & \\
\hline Median (IQR) & $50.50(15.00)$ & $49.00(7.00)$ & $43.00(17.50)$ & $56.50(11.00)$ & \\
\hline
\end{tabular}

${ }^{1}$ Mean \pm SD, Median (IQR) ${ }^{2}$ Kruskal-Wallis rank sum test

Table (3): Distribution of diabetes mellitus and HbA1c in relation to GLS groups

\begin{tabular}{|c|c|c|c|c|c|}
\hline Variable & $\begin{array}{l}\text { Overall, } \\
\mathrm{N}=\mathbf{4 4}^{1}\end{array}$ & $\begin{array}{c}\text { GLS higher than }-10 \\
\& \text { less than }-16, \\
N=17 \\
\end{array}$ & $\begin{array}{c}\text { GLS higher } \\
\text { than }-16 \\
\mathrm{~N}=15 \\
\end{array}$ & $\begin{array}{l}\text { GLS less than }-10, \\
\quad N=12\end{array}$ & p-value ${ }^{2}$ \\
\hline Diabetes Mellitus & & & & & $<0.001$ \\
\hline No & $28(64 \%)$ & $9(53 \%)$ & $15(100 \%)$ & $4(33 \%)$ & \\
\hline Yes & $16(36 \%)$ & $8(47 \%)$ & $0(0 \%)$ & $8(67 \%)$ & \\
\hline HbA1c (\%) & & & & & 0.10 \\
\hline Mean \pm SD & $8.19 \pm 1.14$ & $8.60 \pm 1.07$ & $\mathrm{NA} \pm \mathrm{NA}$ & $7.68 \pm 1.05$ & \\
\hline Median (IQR) & $8.20(2.00)$ & $9.00(1.00)$ & NA (NA) & $7.60(1.52)$ & \\
\hline
\end{tabular}

Table (4): Difference in patients' systolic and diastolic blood pressure (mmHg) across GLS groups

\begin{tabular}{lccccc} 
Variable & $\begin{array}{c}\text { Overall, } \\
\mathbf{N}=\mathbf{4 4}^{\mathbf{1}}\end{array}$ & $\begin{array}{c}\text { GLS higher than - } \\
\mathbf{1 0} \text { \& less than } \mathbf{- 1 6}, \\
\mathbf{N = 1 7}\end{array}$ & $\begin{array}{c}\text { GLS higher } \\
\text { than -16, } \\
\mathbf{N = 1 5}\end{array}$ & $\begin{array}{c}\text { GLS less than -10, } \\
\mathbf{N = 1 2}\end{array}$ & p-value $^{\mathbf{2}}$ \\
\hline $\begin{array}{l}\text { Systolic blood } \\
\text { pressure }\end{array}$ & & & & 0.17 \\
$\quad$ Mean \pm SD & $122.05 \pm 18.37$ & $127.65 \pm 20.16$ & $121.33 \pm 8.34$ & $115.00 \pm 23.16$ & \\
$\quad$ Median (IQR) & $120.00(30.00)$ & $120.00(30.00)$ & $120.00(0.00)$ & $105.00(40.00)$ & \\
\hline $\begin{array}{l}\text { Diastolic blood } \\
\text { pressure }\end{array}$ & & & & & 0.35 \\
$\quad$ Mean \pm SD & $73.41 \pm 12.00$ & $73.53 \pm 13.20$ & $76.00 \pm 10.56$ & $70.00 \pm 12.06$ & \\
$\quad$ Median (IQR) & $70.00(20.00)$ & $70.00(20.00)$ & $70.00(10.00)$ & $65.00(20.00)$ & \\
\hline
\end{tabular}

${ }^{1}$ Mean \pm SD, Median (IQR) ${ }^{2}$ Kruskal-Wallis rank sum test 


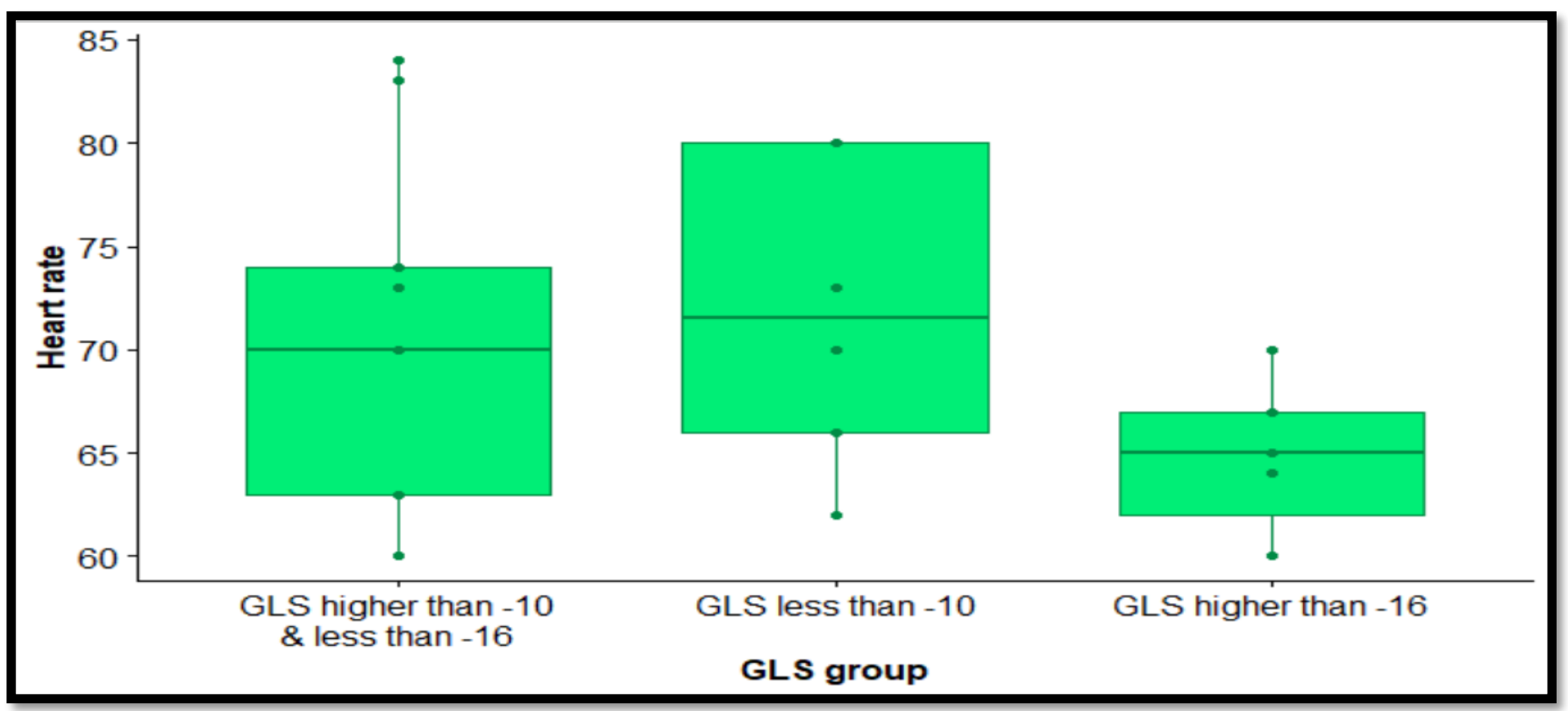

Figure (1): Distribution of heart rate (bpm) across GLS groups

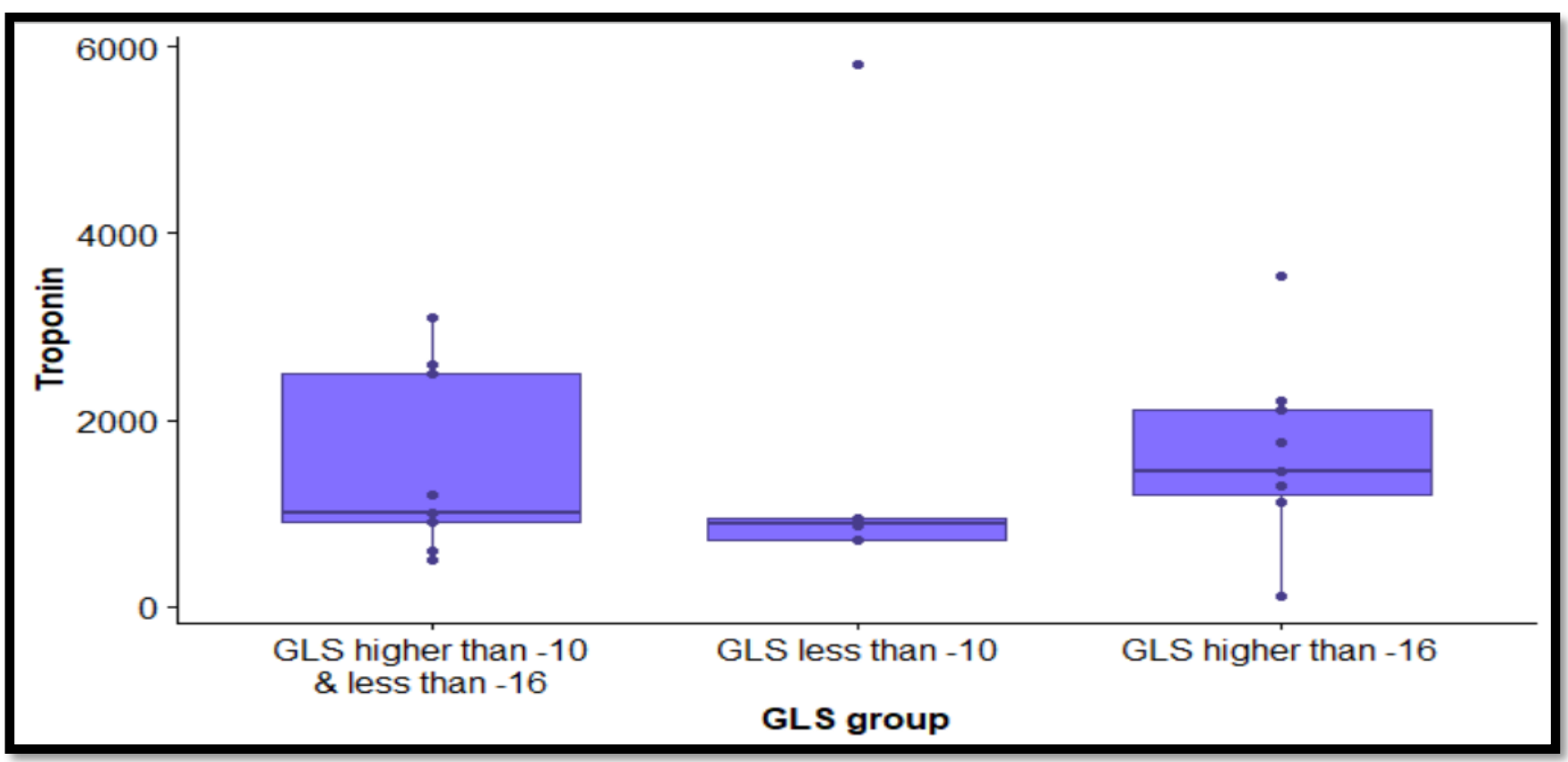

Figure (2): Distribution of troponin across GLS groups

Table (5): Difference in patients' left ventricular ejection fraction across GLS groups

\begin{tabular}{lccccc}
\hline Variable & $\begin{array}{c}\text { Overall, } \\
\mathbf{N}=\mathbf{4 4}^{\mathbf{1}}\end{array}$ & $\begin{array}{c}\text { GLS higher } \\
\text { than -10 \& less } \\
\text { than -16, } \\
\mathbf{N = 1 7}\end{array}$ & $\begin{array}{c}\text { GLS higher } \\
\text { than -16, } \\
\mathbf{N = 1 5}\end{array}$ & $\begin{array}{c}\text { GLS less than - } \\
\mathbf{1 0}, \\
\mathbf{N}=\mathbf{1 2}\end{array}$ & p-value $^{\mathbf{2}}$ \\
\hline LVEF & & & & & $<\mathbf{0 0 1}$ \\
$\quad$ Mean \pm SD & $48.78 \pm 11.30$ & $48.83 \pm 7.42$ & $57.94 \pm 6.42$ & $40.05 \pm 10.35$ & \\
Median (IQR) & $50.60(16.25)$ & $50.30(7.06)$ & $57.00(12.20)$ & $41.23(17.63)$ & \\
\hline
\end{tabular}

${ }^{1}$ Mean \pm SD, Median (IQR) ${ }^{2}$ Kruskal-Wallis rank sum test 


\section{DISCUSSION}

Non-invasive identification of patients with coronary artery disease [CAD] remains a clinical challenge despite the widespread use of imaging and provocative testing; more than $50 \%$ of patients currently referred to coronary angiography show normal or nonobstructive CAD. Severe CAD is known to lead to LV dysfunction. However, the LV ejection fraction is usually normal at a relatively early stage. Thus, establishing a more sensitive index for early-stage ischemia induced LV dysfunction is of great importance. The longitudinally arranged subendocardial fibers are more vulnerable due to their direct exposure to the intraventricular blood pressure and the anatomy of the coronary circulation ${ }^{(13)}$.

As a result, longitudinal function is impaired first in CAD. Measurements of longitudinal motion and deformation are therefore, the most sensitive markers of coronary artery disease especially in patients with severe coronary stenosis, where intermittent ischemia may result in subtle forms of stunning that may be detectable with strain measurements. GLS can be detected by two-dimensional speckle tracking echocardiography (2D-STE) ${ }^{(\mathbf{1 4})}$.

This Prospective cohort study was conducted in Cardiology Department, Faculty of Medicine, Zagazig University. The main aim of this study was to compare between GLS speckle tracking echocardiography and Simpson's biplane methods for assessment of left ventricular function in non-STEMI patients.

As regard socio-demographic data of the studied group; $73 \%$ of the patients in the study were male, $27 \%$ were female. There was a statistically significant difference in gender across GLS groups $(\mathrm{P}=$ 0.004 ). Mean patient age was $50.32 \pm 10.73$ years old. There was a statistically significant difference in age across GLS groups $(\mathrm{P}=0.02)$.

Our results were supported by study of Mostafa et al. $^{\left({ }^{(15)}\right.}$ as they reported that their study included 60 patients with NSTEMI (mean age 54.42 \pm 9.24 years, 38 males and 22 females) and 20 healthy individuals as control group (mean age $36.35 \pm 9.95$ years, 14 males and 6 females). There was statistically significant difference between them regarding age not sex. While, in the study of Liu et al. ${ }^{(16)}$ showed mean age of their study subjects was $58.0 \pm 9.0$ years and $47.5 \%$ were males. No significant differences were observed in age.

The present study showed that $32 \%$ of patients had hypertension. No statistically significant association was found between presence of hypertension and GLS score $(\mathrm{P}=0.14) .36 \%$ of patients had diabetes mellitus, and a statistically significant association was found between presence of diabetes and GLS score $(\mathrm{P}<0.001)$. Mean HbA1c in diabetic patients was $8.19 \pm 1.14 \%$. $27 \%$ of patients were smoker, $18 \%$ were ex-smokers, and $55 \%$ were non- smokers. A statistically significant association was found between smoking and GLS score $(\mathrm{P}<0.001)$.

However, in the study of Kraigher-Krainer $\boldsymbol{e t}$ al. (17) revealed GLS was significantly reduced compared with that in normal controls and was reduced even more in hypertensive patients with heart failure with preserved ejection fraction.

In particular, it seems that longitudinal and radial strain are impaired when circumferential strain is still normal and LV torsion, also maintained in the normal range, acts as mechanistic compensation to preserve a normal ejection fraction $(\mathrm{EF})^{(\mathbf{1 8})}$.

Whereas Madhavan et al. (19) revealed that among the various baseline characteristics, there were no significant differences between the history of past cerebrovascular accident, dyslipidemia, diabetes mellitus. Significant difference was observed in the incidence of hypertension. The patients were categorized in to two groups based on thus obtained mean GLS score [ $\geq-15.7$ and < 15.7].6 months follow up was done and analysis done for MACE based on GRACE score and GLS scores. All the patients who had renal dysfunction were having diabetes and hypertension.

The current study showed mean systolic blood pressure was $122.05 \pm 18.37 \mathrm{mmHg}$. No statistically significant difference in systolic blood pressure was found across GLS groups $(\mathrm{P}=0.17)$. Mean diastolic blood pressure was $73.41 \pm 12.00 \mathrm{mmHg}$. No statistically significant difference in diastolic blood pressure was found across GLS groups $(\mathrm{P}=0.35)$. Mean heart rate was $68.84 \pm 7.13 \mathrm{bpm}$, and a statistically significant difference was found in heart rate across GLS groups $(\mathrm{P}=0.039)$. While, in the study of Liu et al. ${ }^{(16)}$ found no significant differences were observed in heart rate, other risk factors related to the development of atherosclerosis and medical treatment at admission between patients with and without cardiac events.

According to Mostafa et al. ${ }^{(15)}$ showed there were significant differences in clinical features, such as hypertension, diabetes and hyperlipidemia between patients and healthy subjects.

In the study in our hands, mean troponin level was $1524.95 \pm 1226.81 \mathrm{pg} / \mathrm{ml}$. No statistically significant difference was found in troponin level across GLS groups $(\mathrm{P}=0.14)$. Whereas, in the study of Alharbi et al. ${ }^{(20)}$ found there was also a significant association between peak troponin-I level and LVEF ( $\mathrm{r}$ $=-0.557, \mathrm{p}<0.001)$ and LVGLS $(\mathrm{r}=-0.529, \mathrm{p}<$ $0.001)$.

In our study, mean left ventricular ejection fraction was $48.78 \pm 11.30 \%$. A statistically significant difference was found in left ventricular ejection fraction across GLS groups $(\mathrm{P}<0.001)$. In heart failure with preserved ejection fraction there may be reduction in GLS as a sign of reduced systolic function. In patients 
undergoing chemotherapy, the reduction in myocardial strain precedes the significant change in LVEF, and GLS by STE is recommended for early detection of subclinical LV dysfunction ${ }^{(21)}$.

Furthermore, Ersboll et al. ${ }^{(22)}$ demonstrated that 2D global longitudinal strain of the LV was an independent predictor of high risk of developing cardiac events and appeared to be a better parameter than LVEF for prognostic stratification in acute myocardial infarction.

\section{CONCLUSION}

It could be concluded that GLS speckle tracking echocardiography and Simpson's biplane methods can be used as alternative different parameters for assessment of left ventricular function in non STEMI patients.

\section{Financial support and sponsorship: Nil. Conflict of interest: Nil.}

\section{REFERENCES}

1. Potter E, Marwick T (2018): Assessment of left ventricular function by echocardiography: the case for routinely adding global longitudinal strain to ejection fraction. JACC: Cardiovascular Imaging, 11(2 Part 1), 260-274.

2. Roger V, Go A, Lloyd-Jones D et al. (2012): Heart disease and stroke statistics-2012 update: a report from the American Heart Association. Circulation, 125(1): 2-22.

3. Rosamond W, Flegal K, Furie $\mathrm{K}$ et al. (2008): Heart disease and stroke statistics, 2008 update: a report from the American Heart Association Statistics Committee and Stroke Statistics Subcommittee. Circulation, 117(4): 25146.

4. Klaeboe L, Edvardsen T (2019): Echocardiographic assessment of left ventricular systolic function. Journal of Echocardiography, 17(1): 10-16.

5. Ponikowski P, Voors A, Harjola $P$ et al. (2016): ESC guidelines for the diagnosis and treatment of acute and chronic heart failure: the task force for the diagnosis and treatment of acute and chronic heart failure of the European Society of Cardiology (ESC) developed with the special contribution of the heart failure association (HFA) of the ESC. Eur Heart J., 37(27):2129-200.

6. Lang $\mathrm{R}$, Bierig $M$, Devereux $\mathrm{R}$ et al. (2005): Recommendations for chamber quantification: a report from the American Society of Echocardiography's Guidelines and Standards Committee and the Chamber Quantification Writing Group, developed in conjunction with the European Association of Echocardiography, a branch of the European Society of Cardiology. J Am Soc Echocardiogr., 18(12):1440-63.

7. Hoffmann R, Barletta G, von Bardeleben S et al. (2014): Analysis of left ventricular volumes and function: a multicenter comparison of cardiac magnetic resonance imaging, cine ventriculography, and unenhanced and contrast-enhanced two-dimensional and three-dimensional echocardiography. J Am Soc Echocardiogr., 27(3):292-301.

8. Wood P, Choy J, Nanda $\mathbf{N}$ et al. (2014): Left ventricular ejection fraction and volumes: it depends on the imaging method. Echocardiography, 31(1): 87-100.

9. Kalam K, Otahal P, Marwick T (2014): Prognostic implications of global LV dysfunction: a systematic review and meta-analysis of global longitudinal strain and ejection fraction. Heart, 100(21):1673-80.

10. Negishi T, Negishi $K$, Thavendiranathan $P$ et al. (2017): Effect of experience and training on the concordance and precision of strain measurements. JACC Cardiovasc Imaging, 10(5):518-22.

11. Sjoli B, Grenne B, Smiseth $\mathrm{O}$ et al. (2011): The advantage of global strain compared to left ventricular ejection fraction to predict outcome after acute myocardial infarction. Echocardiography, 28(5):556-63.

12. Dean D, Menguç B, Myers C (2000): Revisiting firm characteristics, strategy, and export performance relationship: a survey of the literature and an investigation of New Zealand small manufacturing firms. Industrial Marketing Management, 29: 461-477.

13. Shuvy M, Beeri G, Klein E et al. (2018): Accuracy of the global registry of acute coronary events (GRACE) risk score in contemporary treatment of patients with acute coronary syndrome. Canadian Journal of Cardiology, 34(12): 16131617.

14. Grenne B, Eek C, Dahlslett T et al. (2010): Acute coronary occlusion in non-ST-elevation acute coronary syndrome: outcome and early identification by strain echocardiography. Heart, 96(19):1550-6.

15. Mostafa M, Attia W, Taha M et al. (2018): Assessment of Left Atrial Function in Patients with Non-ST-Segment Elevation Myocardial Infarction Using Two-Dimensional Speckle Tracking Echocardiography. The Egyptian Journal of Hospital Medicine, 73(4):6562-70.

16. Liu C, Jiang S, Li J et al. (2021): Prognostic potential of layer-specific global longitudinal strain in patients with nonST-segment elevated acute coronary syndrome and preserved left ventricular ejection fraction. The International Journal of Cardiovascular Imaging, 37(4):1301-9.

17. Kraigher-Krainer E, Shah A, Pieske B et al. (2014): Impaired systolic function by strain imaging in heart failure with preserved ejection fraction. Journal of the American College of Cardiology, 63(5): 447-456.

18. Wang J, Khoury D, Yue Y et al. (2008): Preserved left ventricular twist and circumferential deformation, but depressed longitudinal and radial deformation in patients with diastolic heart failure. Eur Heart J., 29:1283-1289.

19. Madhavan S, Jayaprasad N, Jayaprakash K et al. (2019): Gender Based Prognostication Value of Left Ventricular Global Longitudinal Strain with Grace Score in non St Elevation Myocardial Infarction A Single Centre Experience. JMSCR., 8: 496-504.

20. Alharbi M, Yahia M, Rashed M et al. (2019): Association between Pentraxin-3 and Cardiac Troponin-I with Left Ventricular Systolic Function in Patients with Anterior STSegment Elevation Myocardial Infarction: Evaluation by Speckle Tracking Echocardiography. World Journal of Cardiovascular Diseases, 9(02):57-62.

21. Plana J, Galderisi M, Barac A et al. (2014): Expert consensus for multimodality imaging evaluation of adult patients during and after cancer therapy: a report from the American Society of Echocardiography and the European Association of Cardiovascular Imaging. Eur Heart J Cardiovasc Imaging, 15:1063-1093.

22. Ersbøll M, Valeur N, Velazquez E et al. (2013): Prediction of all-cause mortality and heart failure admissions from global left ventricular longitudinal strain in patients with acute myocardial infarction and preserved left ventricular ejection fraction. Journal of the American College of Cardiology, 61(23):2365-73. 